\title{
Performance Study of Wireless Systems with Switch and Stay Combining Diversity over $\alpha-\eta-\mu$ Fading Channels
}

\author{
Ali A. Eyadeh \\ Communication Engineering Department \\ Yarmouk University \\ Irbid, Jordan \\ aeyadeh@yu.edu.jo
}

\author{
Mohammad N. Al-Ta'ani \\ Communication Engineering Department \\ Yarmouk University \\ Irbid, Jordan \\ mohd.taani89@gmail.com
}

\begin{abstract}
In this paper, we consider a Switch and Stay Combiner (SSC) diversity scheme operating over $\alpha-\eta-\mu$ fading channel. New and closed-form expressions for the average output SNR (ASNR), the moment-generating function (MGF), the outage probability (P_out), and the average symbol error rate (ASER) for M-ary quadrature amplitude modulation (QAM) signaling are derived. The expressions are obtained in terms of the wellknown bivariate Fox's H-function (BFHF). It is worth pointing out that the BFHF and the bivariate Meijer's G-function (BMGF) have recently been used extensively in wireless communications literature to study the system's performance. The evaluated results are plotted for channel parameters of interest, and the effect of fading severity on the combiner performance is studied. Moreover, the results are shown to match those previously reported in the literature for other channel models such as $\eta-\mu$ as a special case, which confirms the validity of the obtained expressions. Also, insights on the optimal choice of the switching threshold are provided.
\end{abstract}

Keywords-switch and stay combiner SSC diversity; $\alpha-\eta-\mu$ fading channel; M-ary QAM; average output SNR (ASNR); moment-generating function $(M G F)$; outage probability $\left(P_{\text {out }}\right)$

\section{INTRODUCTION}

There are many distributions that describe properly the statistics of the mobile radio signal. The long-term signal variation is acknowledged to follow the lognormal distribution, whereas the short-term signal variation is described by various other distributions such as Hoyt, Rayleigh, Rice, Nakagami-m, and Weibull. It is typically accepted that the path strength at any extend is characterized by short-term distributions over a spatial dimension of a few hundred wavelengths, and with the aid of the lognormal distribution over areas with larger dimensions. The $\alpha-\eta-\mu$ distribution is a generic fading distribution used to signify the small-scale variation of the fading signal. Channel multipath fading is an important consideration when designing a wireless communication system, therefore fading mitigation techniques are needed. Diversity combining is an effective technique used to mitigate fading and improves the performance of wireless systems over a fading channel. There are various types of diversity combining techniques used in practice [1], for example
Selection Combining (SC), Equal Gain Combining (EGC), Maximal Ratio Combining (MRC) and Switched Diversity Combining (SDC). Two strategies can be used in SDC: Switch and Stay Combining (SSC) which is considered in this paper, and Switch and Examine Combining (SEC). In an SSC diversity system, the receiver selects a branch until its signalto-noise ratio (SNR) drops below a predetermined threshold. When this happens, the combiner switches to another branch and stays there regardless of whether the SNR of the original branch is above or below the predetermined threshold.

Several works have been conducted on the analysis of SSC scheme over fading channels including [2-14]. In [2-5], the performance of SSC for non-coherent binary frequency shift keying (BFSK) and noncoherent M-ary frequency shift keying (MFSK) over correlated Nakagami-m and Rician fading channels was studied. In [6], the performance of non-coherent MFSK with selection and switched diversity was analyzed over a Hoyt fading channel. The performance of correlated Rician fading channels and correlated Weibull fading channels with SSC diversity was evaluated in [7] and [8] respectively. The performance of a dual-branch SSC systems over Nakagami-m, correlated $\alpha-\mu$, correlated $\eta-\mu$ and correlated generalized-K (KG) fading channels were studied and analyzed in [9-14]. Popular fading distributions have been derived assuming a homogeneous diffuse scattering field, resulting from randomly distributed point scatters. The assumption of a homogeneous field is truly an approximation because the surfaces are spatially correlated characterizing a non-linear environment. With the aim at exploring this non-homogeneity, two new fading distributions, $\kappa-\mu$ and $\eta-\mu$ have been discussed in [15, $16]$, and to discover the non-linearity of the propagation medium, which was also addressed more currently in a new proposed common fading distribution, the $\alpha-\mu$ distribution [17].

The $\alpha-\eta-\mu$ distribution is an accepted distribution for a short-time fading model. The probability density function (PDF) of $\alpha-\eta-\mu$ distribution is in the shape of three parameters $\alpha, \eta$ and $\mu$, which are associated to the nonlinearity of the environment, the scattered wave power ratio between the inphase, and quadrature components of each cluster of multipath 
and the number of multipath clusters in the environment respectively. The $\alpha-\eta-\mu$ model includes, as special cases, other short-time fading distributions, like Rayleigh, Nakagami- $m$, Nakagami- $q$ (Hoyt), Weibull, $\eta-\mu$ and One-Side Gaussian distribution. By setting $\alpha=2$, it reduces to $\eta-\mu$ distribution. Furthermore, from $\eta-\mu$ fading distribution, Nakagami- $m$ model could be obtained in two cases: first for $\eta \rightarrow 1$, with Nakagami parameter $m$ being expressed as $\mu=m / 2$, and second for $\eta \rightarrow 0$, with parameter $\mathrm{m}$ being expressed as $\mu=m$. It is well-known that $\eta-\mu$ distribution reduces to Hoyt distribution, when $\mu=1$, with Hoyt parameter $q$ defined as $q=(1-\eta) /(1+\eta)$. From the Hoyt distribution the One-Sided Gaussian is obtained for $q \rightarrow+1$ or $q \rightarrow-1(\eta \rightarrow 0$ or $\eta \rightarrow \infty)$. In the same way, by equating the in-phase and quadrature components variances, namely by setting $\eta=1$, Rayleigh distribution is derived from Hoyt. Also, Weibull distribution could be obtained as a special case of the $\alpha-\eta-\mu$ model by setting corresponding values to the parameters $\mu=1$ and $\eta=1$. In [18], $\alpha-\eta-\mu$ and $\alpha-\kappa-\mu$ distributions have been discussed. The performance analysis of wireless communication over $\alpha-\eta-\mu$ fading channel has been investigated in [19], where the outage probability, PDF and $\mathrm{CDF}$ of the received signal to interference ratio were derived. The performance analysis of $\alpha-\eta-\mu$ fading channel is carried out in [20], when the communication is subjected to influence of co-channel interference. In [21], the performance of digital communication systems that operate over $\alpha-\eta-\mu$ fading channels was analyzed and evaluated. Specifically, exact closed-form analytical expressions for MGF, CDF, average channel capacity, and ASEP for different coherent and noncoherent modulation schemes were derived.

Switched diversity combining schemes such as SSC are less complex diversity combining schemes, as SSC does not require channel estimation at the receiver, and minimizes the switching rate required the available diversity branches. Though this diversity combining scheme was already examined over different fading channels including Rayleigh, Rician, Nakagami, etc., analysis over fading distributions such as $\alpha-\eta-\mu$ is not available in the literature. In this paper, we derived novel and closed-form expressions for ASNR, outage probability (Pout), MGF, and the ASER for the M-ary quadrature amplitude modulation (QAM) scheme, of a dual-branch SSC operating over generalized $\alpha-\eta-\mu$ fading channels. The expressions are obtained in terms of the bivariate Fox's Hfunction (BFHF). It is worth pointing out that the BFHF and the bivariate Meijer's G-function (BMGF) have recently been used extensively in the wireless communications literature when studying the systems' performance. The evaluated results are plotted for channel parameters of interest, and the effect of fading severity on the combiner performance is studied. Our derived expressions are valid for arbitrary values of the fading parameters $\alpha, \eta$ and $\mu$. Other short-time fading distributions, like Rayleigh, Nakagami-m, Nakagami-q (Hoyt), Weibull, $\eta-\mu$ and One-Side Gaussian distribution, are derived from our results as special cases.

\section{SYSTEM MODEL AND OUTPUT STATISTICS}

In this paper, we focus on the performance evaluation of SSC systems over $\alpha-\eta-\mu$ fading channels. In these dual-branch diversity systems the receiver selects a branch until its SNR drops below a predetermined threshold. When this happens, the combiner switches to another branch and stays there regardless of whether the SNR of the original branch is above or below the predetermined threshold.

\section{A. The $\alpha-\eta-\mu$ Distribution}

\section{1) Probability Density Function:}

We assume that the channel envelope R follows the $\alpha-\eta-\mu$ distribution. The probability density function (PDF) of the channel under consideration is given as [22]:

$$
f_{R}(r)=\frac{2 \sqrt{\pi} \alpha h^{\mu} \mu^{\mu+\frac{1}{2} r^{\alpha\left(\mu+\frac{1}{2}\right)-1} I_{\mu-\frac{1}{2}}\left(\frac{2 \mu H r^{\alpha}}{\bar{r}^{\alpha}}\right)}}{\Gamma(\mu) H^{\mu-\frac{1}{2} \bar{r}^{\alpha}\left(\mu+\frac{1}{2}\right)} \exp \left(\frac{2 \mu h r^{\alpha}}{\bar{r}^{\alpha}}\right)}
$$

where $\Gamma(z)=\int_{0}^{\infty} t^{z-1} \exp (-t) \mathrm{d} t$ is the Gamma function, $I_{v}(\cdot)$ is the modified Bessel function of the first kind and arbitrary order $v, \alpha>0, \mu>0, h=\frac{(1+\eta)^{2}}{4 \eta}, H=\frac{\left(1-\eta^{2}\right)}{4 \eta}, \eta>0$, and $\bar{r}^{\alpha}$ represents the $\alpha$-root mean value of the envelope $r$. To derive the cumulative density function (CDF) of the SNR, we need first to derive the PDF for the SNR. As such, we define the instantaneous SNR, $\gamma$, as [23]:

$$
\gamma=\bar{\gamma}\left(\frac{r}{\bar{r}}\right)^{2}
$$

where $\bar{\gamma}=E\left[\bar{r}^{2}\right] E_{b} / N_{o}$ and $E_{b} / N_{o}$ is the energy per bit to the noise power spectral density ratio. After performing random variable transformation using (1) and (2) [24], then the PDF of $\gamma$ is obtained as:

$$
f_{\gamma}(\gamma)=\frac{\sqrt{\pi} \alpha h^{\mu} \mu^{\mu+\frac{1}{2}} \gamma^{\frac{\alpha}{2}\left(\mu+\frac{1}{2}\right)-1} I_{\mu-\frac{1}{2}}\left(\frac{2 \mu H \gamma^{\frac{\alpha}{2}}}{\bar{\gamma}^{\frac{\alpha}{2}}}\right)}{\Gamma(\mu) H^{\mu-\frac{1}{2}} \bar{\gamma}^{\frac{\alpha}{2}\left(\mu+\frac{1}{2}\right)} \exp \left(\frac{2 \mu h \gamma^{\frac{\alpha}{2}}}{\bar{\gamma}^{\frac{\alpha}{2}}}\right)}
$$

The unified PDF defined in (3) can be written in terms of the Fox's H-function as [21]:

$$
\begin{gathered}
f_{\gamma}(\gamma)=\frac{\alpha h^{\mu} \mu^{\mu+\frac{1}{2}} \gamma^{\frac{\alpha}{2}\left(\mu+\frac{1}{2}\right)-1}}{\Gamma(\mu) H^{\mu-\frac{1}{2}} \bar{\gamma}^{\frac{\alpha}{2}\left(\mu+\frac{1}{2}\right)}} \\
\times H_{0,1}^{1,0}\left[\frac{2 \mu(h-H) \gamma^{\frac{\alpha}{2}}}{\bar{\gamma}^{\frac{\alpha}{2}}} \mid \begin{array}{c}
- \\
(0,1)
\end{array}\right] H_{1,2}^{1,1}\left[\frac{4 \mu H \gamma^{\frac{\alpha}{2}}}{\bar{\gamma}^{\frac{\alpha}{2}}} \mid \begin{array}{c}
\left(\frac{1}{2}, 1\right) \\
\left(\mu-\frac{1}{2}, 1\right),\left(\frac{1}{2}-\mu, 1\right)
\end{array}\right]
\end{gathered}
$$

where $H_{p, q}^{m, n}[$.$] is the Fox's H-function [25].$

\section{2) The Cumulative Distribution Function}

The CDF of $\gamma$ with its corresponding PDF defined in (3) can be derived using the main definition of the CDF, which is given by [1] as:

$$
F_{\gamma}=\int_{0}^{\gamma} f_{\gamma}(\xi) d \xi
$$

For arbitrary values of $\mu$, the CDF of $\gamma$ in (5) can be expressed as [18]:

$$
F_{\gamma}(\gamma)=1-Y_{\mu}\left(\frac{H}{h}, \sqrt{\frac{2 \mu h \gamma^{\frac{\alpha}{2}}}{\bar{\gamma}^{\frac{\alpha}{2}}}}\right)
$$


where

$$
Y_{\mu}(a, b)=\frac{\sqrt{\pi} 2^{\frac{3}{2}-\mu}\left(1-a^{2}\right)^{\mu}}{\Gamma(\mu) a^{\mu-\frac{1}{2}}} \int_{\mathrm{b}}^{\infty} x^{2 \mu} I_{\mu-\frac{1}{2}}\left(a x^{2}\right) \exp \left(-x^{2}\right) d x
$$

denotes the Yacoub integral [15]. Hence, the CDF of $\gamma$ is obtained as:

$$
\begin{gathered}
F_{\gamma}(\gamma)= \\
\frac{\left(1-\left(\frac{H}{h}\right)^{2}\right)^{\mu}}{\Gamma(2 \mu+1)}\left(2 \mu h\left(\frac{\gamma}{\bar{\gamma}}\right)^{\frac{\alpha}{2}}\right)^{2 \mu} \\
\times \Phi_{2}\left[\mu, \mu ; 2 \mu+1 ;-\left(1 \frac{H}{h}\right)\left(2 \mu h\left(\frac{\gamma}{\bar{\gamma}}\right)^{\frac{\alpha}{2}}\right),-\left(-\frac{H}{h}\right)\left(2 \mu h\left(\frac{\gamma}{\bar{\gamma}}\right)^{\frac{\alpha}{2}}\right)\right]
\end{gathered}
$$

where $\Phi_{2}$ is the confluent Lauricella function [26].

\section{3) The Moment Generating Function (MGF)}

The MGF for the $\alpha-\eta-\mu$ fading channels is obtained using the BFHF as [21]:

$$
\begin{aligned}
& M_{\gamma}(s)=\frac{\alpha h^{\mu} \mu^{\mu+\frac{1}{2}}}{\Gamma(\mu) H^{\mu-\frac{1}{2}}(s \bar{\gamma})^{\frac{\alpha}{2}\left(\mu+\frac{1}{2}\right)}} \times \mathrm{H}_{1,0: 0,1 ; 1,2}^{0,1: 1,0 ; 1,} \\
& {\left[\begin{array}{c|c}
\frac{2 \mu(h-H)}{(s \bar{\gamma}) \frac{\alpha}{2}} & \left(1-\frac{\alpha}{2}\left(\mu+\frac{1}{2}\right) ; \frac{\alpha}{2}, \frac{\alpha}{2}\right):-;\left(\frac{1}{2}, 1\right) \\
\frac{4 \mu H}{(s \bar{\gamma})^{\frac{\alpha}{2}}} & -:(0,1) ;\left(\mu-\frac{1}{2}, 1\right),\left(\frac{1}{2}-\mu, 1\right)
\end{array}\right]}
\end{aligned}
$$

\section{B. Output Statistics of the SSC System:}

The PDF, CDF and MGF of the received SNR at the output of a dual-branch SSC system over $\alpha-\eta-\mu$ fading channels are derived in this section.

\section{1) The PDF of the Received $S N R$}

If $\gamma s S c$ denotes the SNR at the output of an SSC combiner, and $\gamma_{T}$ denotes the predetermined switching threshold, to derive the PDF of SSC of the output SNR, we first derive the CDF of the output SNR, $F_{\gamma s s c}(\gamma)$, in terms of CDF of individual branch SNR, $F_{\gamma}(\gamma)$, as [1]:

$$
F_{\gamma s s c}(\gamma)=\left\{\begin{array}{ll}
F_{\gamma}\left(\gamma_{T}\right) F_{\gamma}(\gamma) & , \gamma<\gamma_{T} \\
F_{\gamma}(\gamma)-F_{\gamma}\left(\gamma_{T}\right)+F_{\gamma}\left(\gamma_{T}\right) F_{\gamma}(\gamma), & \gamma \geq_{\gamma_{T}}
\end{array}(9)\right.
$$

Therefore, the CDF for a dual branch $\alpha-\eta-\mu$ fading channels with SSC diversity is obtained by inserting (7) into (9) as:

$$
F_{\gamma s s c}(\gamma)= \begin{cases}\boldsymbol{A} F_{\gamma}(\gamma) & , \gamma<\gamma_{T} \\ F_{\gamma}(\gamma)-\boldsymbol{A}+\boldsymbol{A} F_{\gamma}(\gamma), & \gamma \geq_{\gamma_{T}}\end{cases}
$$

where

$$
\begin{gathered}
\boldsymbol{A}=F_{\gamma}\left(\gamma_{T}\right)= \\
\frac{\left.\left(1-\frac{H}{h}\right)^{2}\right)^{\mu}}{\Gamma(2 \mu+1)}\left(2 \mu h\left(\frac{\gamma_{T}}{\bar{\gamma}}\right)^{\frac{\alpha}{2}}\right)^{2 \mu} \\
\times \Phi_{2}\left[\mu, \mu ;+1 ;-\left(1+\frac{H}{h}\right)\left(2 \mu h\left(\frac{\gamma_{T}}{\bar{\gamma}}\right)^{\frac{\alpha}{2}}\right),-\left(1-\frac{H}{h}\right)\left(2 \mu h\left(\frac{\gamma_{T}}{\bar{\gamma}}\right)^{\frac{\alpha}{2}}\right)\right]
\end{gathered}
$$

Differentiating $F_{\gamma s s c}(\gamma)$ with respect to $\gamma$, we get the PDF of the SNR at the output of the SSC combiner, $f_{\gamma s s c}(\gamma)$, in terms of $\operatorname{CDF} F_{\gamma}(\gamma)$,and the PDF $f_{\gamma}(\gamma)$ of the individual branch SNR as [1]:

$$
f_{\gamma s s c}(\gamma)=\frac{d F_{\gamma s s}(\gamma)}{d \gamma}= \begin{cases}F_{\gamma}\left(\gamma_{T}\right) f_{\gamma}(\gamma), & \gamma<\gamma_{T} \\ {\left[1+F_{\gamma}\left(\gamma_{T}\right)\right] f_{\gamma}(\gamma),} & \gamma \geq_{\gamma_{T}}\end{cases}
$$

The PDF $f_{\gamma s s c}(\gamma)$, for fading channels with SSC diversity is found by inserting (7) with respect to $\gamma_{T}$, and one of the PDF expressions (3) or (4) into (11) as:

$$
f_{\gamma s s c}(\gamma)= \begin{cases}\boldsymbol{A} f_{\gamma}(\gamma) & , \gamma<\gamma_{T} \\ {[1+\boldsymbol{A}] f_{\gamma}(\gamma)} & , \gamma \geq_{\gamma_{T}}\end{cases}
$$

where $\boldsymbol{A}=F_{\gamma}\left(\gamma_{T}\right)$ as mentioned previously in (10).

\section{2) The MGF of the Received $S N R$}

The MGF with SSC diversity receiver, operating over $\alpha-\eta-\mu$ fading channels is obtained using the PDF in (12) that is [1]:

$$
\begin{gathered}
M_{\gamma s s c}(s)=\int_{0}^{\infty} \mathrm{e}^{-s \gamma} f_{\gamma s s c}(\gamma) d \gamma \\
=\left[1+F_{\gamma}\left(\gamma_{T}\right)\right] M_{\gamma}(s)-\underbrace{\int_{0}^{\gamma_{T}} \mathrm{e}^{-s \gamma} f_{\gamma}(\gamma) d \gamma}_{I_{1}}
\end{gathered}
$$

where $M_{\gamma}(s)$ is the MGF for an individual branch under $\alpha-\eta-\mu$ fading channel which is derived in (8). To obtain an expression for $M_{\gamma s s c}(s)$ in (13), we need to solve the second term integral $I_{1}$. To calculate the integral $I_{1}$ in (13) we insert (4) in $I_{1}$ as:

$$
\begin{aligned}
& I_{1}=\frac{\alpha h^{\mu} \mu^{\mu+\frac{1}{2}}}{\Gamma(\mu) H^{\mu-\frac{1}{2}} \bar{\gamma}^{\frac{\alpha}{2}\left(\mu+\frac{1}{2}\right)}} \int_{0}^{\gamma_{T}} \gamma^{\frac{\alpha}{2}\left(\mu+\frac{1}{2}\right)-1} \\
& \times \mathrm{H}_{0,1}^{1,0}\left[\frac{2 \mu(h-H) \gamma^{\frac{\alpha}{2}}}{\bar{\gamma}^{\frac{\alpha}{2}}} \mid \begin{array}{c}
- \\
(0,1)
\end{array}\right] \mathrm{H}_{1,2}^{1,1}\left[\frac{4 \mu H \gamma^{\frac{\alpha}{2}}}{\bar{\gamma}^{\frac{\alpha}{2}}} \mid \begin{array}{c}
\left(\frac{1}{2}, 1\right) \\
\left(\mu-\frac{1}{2}, 1\right),\left(\frac{1}{2}-\mu, 1\right)
\end{array}\right] \\
& \times \mathrm{H}_{0,1}^{1,0}\left[\left.s \gamma\right|_{(0,1)} ^{-}\right] d \gamma
\end{aligned}
$$

Note that (14) is found by expressing the exponential function in $I_{1}$, in terms of the Fox's $H$ function [25]. Using the definition of Fox's H function [25], (14) is written as:

$$
\begin{aligned}
& I_{1}=\frac{\alpha h^{\mu} \mu^{\mu+\frac{1}{2}}}{\Gamma(\mu) H^{\mu-\frac{1}{2} \bar{\gamma}^{\frac{\alpha}{2}}\left(\mu+\frac{1}{2}\right)}}\left(\frac{1}{2 \pi i}\right)^{3} \\
& \times \int_{L_{1}} \int_{L_{2}} \int_{L_{3}} \Gamma\left(\xi_{1}\right)\left(\frac{2 \mu(h-H)}{\bar{\gamma}^{\frac{\alpha}{2}}}\right)^{-\xi_{1}} \\
& \times \frac{\Gamma\left(\mu-\frac{1}{2}+\xi_{2}\right) \Gamma\left(\frac{1}{2}-\xi_{2}\right)}{\Gamma\left(\frac{1}{2}+\mu-\xi_{2}\right)}\left(\frac{4 \mu H}{\bar{\gamma}^{\frac{\alpha}{2}}}\right)^{-\xi_{2}} \Gamma\left(\xi_{3}\right)(s)^{-\xi_{3}} \\
& \times[\underbrace{\int_{0}^{\gamma_{T}} \gamma^{\frac{\alpha}{2}\left(\mu+\frac{1}{2}-\xi_{1}-\xi_{2}\right)-\xi_{3}-1} d \gamma}_{\widetilde{I_{1}}}] d \xi_{1} d \xi_{2} d \xi_{3}
\end{aligned}
$$

The inner integral in (15) with respect to $\gamma\left(\right.$ i.e., $\overline{I_{1}}$ ) can be solved using the power integration rule:

$$
\overline{I_{1}}=\int_{0}^{\gamma_{T}} \gamma^{\frac{\alpha}{2}\left(\mu+\frac{1}{2}-\xi_{1}-\xi_{2}\right)-\xi_{3}-1} d \gamma
$$




$$
=\frac{\gamma_{T}^{\frac{\alpha}{2}}\left(\mu+\frac{1}{2}-\xi_{1}-\xi_{2}\right)-\xi_{3}}{\frac{\alpha}{2}\left(\mu+\frac{1}{2}-\xi_{1}-\xi_{2}\right)-\xi_{3}}
$$

By using the identity $\Gamma(x+1)=x \Gamma(x)$ [27], then (16) can be written as:

$$
\overline{I_{1}}=\frac{\gamma_{T}^{\frac{\alpha}{2}\left(\mu+\frac{1}{2}-\xi_{1}-\xi_{2}\right)-\xi_{3}} \Gamma\left(\frac{\alpha}{2}\left(\mu+\frac{1}{2}-\xi_{1}-\xi_{2}\right)-\xi_{3}\right)}{\Gamma\left(\frac{\alpha}{2}\left(\mu+\frac{1}{2}-\xi_{1}-\xi_{2}\right)-\xi_{3}+1\right)}
$$

Now, substituting (17) into (15) yields:

$$
\begin{gathered}
I_{1}=\frac{\alpha h^{\mu} \mu^{\mu+\frac{1}{2}} \gamma_{T} \frac{\alpha}{2}\left(\mu+\frac{1}{2}\right)}{\Gamma(\mu) H^{\mu-\frac{1}{2}} \frac{\alpha}{2}\left(\mu+\frac{1}{2}\right)}\left(\frac{1}{2 \pi i}\right)^{3} \\
\times \int_{L_{1}} \int_{L_{2}} \int_{L_{3}} \Gamma\left(\xi_{1}\right)\left(\frac{2 \mu(h-H) \gamma_{T} \frac{\alpha}{2}}{\bar{\gamma}^{\frac{\alpha}{2}}}\right)^{-\xi_{1}} \\
\times \frac{\Gamma\left(\mu-\frac{1}{2}+\xi_{2}\right) \Gamma\left(\frac{1}{2}-\xi_{2}\right)}{\Gamma\left(\frac{1}{2}+\mu-\xi_{2}\right)}\left(\frac{4 \mu H \gamma_{T} \frac{\alpha}{2}}{\bar{\gamma}^{\frac{\alpha}{2}}}\right)^{-\xi_{2}} \Gamma\left(\xi_{3}\right)\left(s \gamma_{T}\right)^{-\xi_{3}} \\
\times \frac{\Gamma\left(\frac{\alpha}{2}\left(\mu+\frac{1}{2}-\xi_{1}-\xi_{2}\right)-\xi_{3}\right)}{\Gamma\left(\frac{\alpha}{2}\left(\mu+\frac{1}{2}-\xi_{1}-\xi_{2}\right)-\xi_{3}+1\right)} d \xi_{1} d \xi_{2} d \xi_{3}
\end{gathered}
$$

Using A.1 from [28], then the inner integral $I_{1}$ is found in terms of the BFHF as:

$$
\begin{gathered}
I_{1}=\frac{\alpha h^{\mu} \mu^{\mu+\frac{1}{2}} \gamma_{T} \frac{\alpha}{2}\left(\mu+\frac{1}{2}\right)}{\Gamma(\mu) H^{\mu-\frac{1}{2}} \frac{\alpha}{2}\left(\mu+\frac{1}{2}\right)} \times \mathrm{H}_{1,1: 0,1 ; 1,2 ; 0,1}^{0,1: 1,0 ; 1,1 ; 1,0} \\
{\left[\begin{array}{cc}
\frac{2 \mu(h-H) \gamma_{T} \frac{\alpha}{2}}{\bar{\gamma}^{\frac{\alpha}{2}}} & \left(1-\frac{\alpha}{2}\left(\mu+\frac{1}{2}\right) ; \frac{\alpha}{2}, \frac{\alpha}{2}, 1\right):-;\left(\frac{1}{2}, 1\right) ;- \\
\frac{4 \mu H \gamma_{T}^{\frac{\alpha}{2}}}{\frac{\alpha}{\frac{\alpha}{2}}} & \left(-\frac{\alpha}{2}\left(\mu+\frac{1}{2}\right) ; \frac{\alpha}{2}, \frac{\alpha}{2}, 1\right):(0,1) ;\left(\mu-\frac{1}{2}, 1\right),\left(\frac{1}{2}-\mu, 1\right) ;(0,1) \\
S \gamma_{T} &
\end{array}\right]}
\end{gathered}
$$

In conclusion, a closed form expression of the $M_{\gamma s s c}(s)$, is obtained as:

$$
\begin{aligned}
& M_{\gamma s S C}(s)=\left(1+F_{\gamma}\left(\gamma_{T}\right)\right) \frac{\alpha h^{\mu} \mu^{\mu+\frac{1}{2}}}{\Gamma(\mu) H^{\mu-\frac{1}{2}}(s \bar{\gamma})^{\frac{\alpha}{2}\left(\mu+\frac{1}{2}\right)}}
\end{aligned}
$$

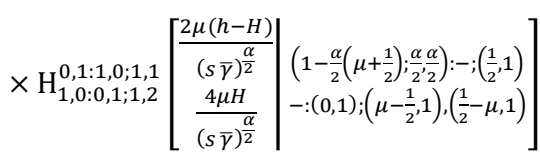

$$
\begin{aligned}
& -\frac{\alpha h^{\mu} \mu^{\mu+\frac{1}{2}} \gamma_{T^{\frac{\alpha}{2}}}\left(\mu+\frac{1}{2}\right)}{\Gamma(\mu) H^{\mu-\frac{1}{2} \bar{\gamma}^{\frac{\alpha}{2}}\left(\mu+\frac{1}{2}\right)}} \times \mathrm{H}_{1,1: 0,1 ; 1,2 ; 0,1}^{0,1: 0 ; 1,1 ; 1,0} \\
& {\left[\begin{array}{cc}
\frac{2 \mu(h-H) \gamma_{T} \frac{\alpha}{2}}{\bar{\gamma}^{\frac{\alpha}{2}}} & \left(1-\frac{\alpha}{2}\left(\mu+\frac{1}{2}\right) ; \frac{\alpha}{2}, \frac{\alpha}{2}, 1\right):-;\left(\frac{1}{2}, 1\right) ;- \\
\frac{4 \mu H \gamma_{T}^{\frac{\alpha}{2}}}{\bar{\gamma}^{\frac{\alpha}{2}}} & \left(-\frac{\alpha}{2}\left(\mu+\frac{1}{2}\right) ; \frac{\alpha}{2}, \frac{\alpha}{2}, 1\right):(0,1) ;\left(\mu-\frac{1}{2}, 1\right),\left(\frac{1}{2}-\mu, 1\right) ;(0,1) \\
S \gamma_{T} &
\end{array}\right]}
\end{aligned}
$$

\section{PERFORMANCE ANALYSIS OF THE SSC SYSTEM}

Many important measures characterize the performance of communication systems in fading environments, such as the average symbol error probability (ASEP), the ASNR or the $P_{\text {out }}$, can be determined by averaging appropriate performance functions over the distribution of the effective SNR at the receiver-side. In this section a detailed performance analysis, in terms of $P_{\text {out }}$, ASEP and ASNR, for SSC diversity receivers operating over $\alpha-\eta-\mu$ fading channels is presented.

\section{A. Outage Probability}

The outage probability is the probability that the SNR at the output of the SSC falls below a threshold level, $\gamma_{t h}$, which is found by replacing $\gamma$ in $F_{\gamma s s c}(\gamma)$ with $\gamma_{t h}$ as in 9.241 in [1]:

$$
P_{\text {out }}^{\text {ssc }}\left(\gamma_{t h}\right)=P_{r}\left[\gamma_{s s c} \leq \gamma_{t h}\right]=F_{\gamma s s c}\left(\gamma_{t h}\right)
$$

Since the SDC is considered as an optimal implementation of the switched diversity system, then the optimal switching threshold in the minimum outage probability is: $\gamma_{T, o p t}=\gamma_{t h}$, and because the outage probability of a dual-branch $\mathrm{SC}$ is $P_{\text {out }}^{s c}\left(\gamma_{t h}\right)=\left[F_{\gamma}\left(\gamma_{t h}\right)\right]^{2}[1]$, then the outage probability of a dual-branch SSC system with an optimal switching threshold is [1]:

$$
P_{\text {out }}^{s s c}\left(\gamma_{t h}\right)=P_{\text {out }}^{s c}\left(\gamma_{t h}\right)=\left[F_{\gamma}\left(\gamma_{t h}\right)\right]^{2}
$$

where $F_{\gamma}\left(\gamma_{t h}\right)$ is (7) after replacing $\gamma$ with $\gamma_{t h}$. As a result, a closed form expression of the $P_{\text {out }}^{s s c}\left(\gamma_{t h}\right)$ is obtained after inserting (7), after replacing $\gamma$ with $\gamma_{t h}$, into (22) as:

$$
\begin{gathered}
P_{\text {out }}^{\text {ssc }}\left(\gamma_{\text {th }}\right)=\frac{\left(1-\left(\frac{H}{h}\right)^{2}\right)^{2 \mu}}{(\Gamma(2 \mu+1))^{2}}\left(2 \mu h\left(\frac{\gamma_{\text {th }}}{\bar{\gamma}}\right)^{\frac{\alpha}{2}}\right)^{4 \mu} \\
\times\left(\Phi_{2}\left[\mu, \mu ; 2 \mu+1 ;-\left(1+\frac{H}{h}\right)\left(2 \mu h\left(\frac{\gamma_{t h}}{\bar{\gamma}}\right)^{\frac{\alpha}{2}}\right),-\left(1-\frac{H}{h}\right)\left(2 \mu h\left(\frac{\gamma_{t h}}{\bar{\gamma}}\right)^{\frac{\alpha}{2}}\right)\right]\right)^{2}
\end{gathered}
$$

\section{B. Average Output SNR}

The average SNR at the SSC output, $\bar{\gamma}_{s S c}$, is a useful performance measure serving as an excellent indicator for the overall system fidelity, and it can be obtained by averaging $\gamma$ over $f_{\gamma s s c}(\gamma)[1]$ :

$$
\bar{\gamma}_{s S c}=\int_{0}^{\infty} \gamma f_{\gamma s s c}(\gamma) d \gamma=\left[1+F_{\gamma}\left(\gamma_{T}\right)\right] \bar{\gamma}-\underbrace{\int_{0}^{\gamma_{T}} \gamma f_{\gamma}(\gamma) d \gamma}_{I_{2}}
$$

Differentiating (24) with respect to $\gamma_{T}$ and setting the result to zero, it can be easily shown that $\bar{\gamma}_{s s c}$ is maximized when the switching threshold is set to $\gamma_{T, o p t}=\bar{\gamma}$. To obtain an expression for $\bar{\gamma}_{s S c}$ in (24), we need to solve the second term integral $I_{2}$. Following similar steps as in (13) and using 2.57 from [28], $I_{2}$ is found in terms of the BFHF as:

$$
I_{2}=\frac{\alpha h^{\mu} \mu^{\mu+\frac{1}{2}} \gamma_{T}^{\frac{\alpha}{2}\left(\mu+\frac{1}{2}\right)+1}}{\Gamma(\mu) H^{\mu-\frac{1}{2}} \bar{\gamma}^{\frac{\alpha}{2}\left(\mu+\frac{1}{2}\right)}}
$$

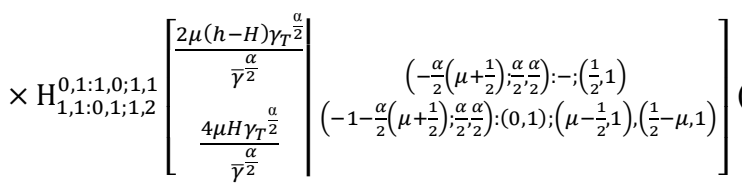

Finally, a closed form expression of the $\bar{\gamma}_{S S C}$ is obtained as:

$$
\bar{\gamma}_{S S C}=\left(\bar{\gamma}+\bar{\gamma} F_{\gamma}\left(\gamma_{T}\right)\right)-\frac{\alpha h^{\mu} \mu^{\mu+\frac{1}{2}} \gamma_{T^{\frac{1}{2}}}^{\frac{\alpha}{2}\left(\mu+\frac{1}{2}\right)+1}}{\Gamma(\mu) H^{\mu-\frac{1}{2}} \bar{\gamma}^{\frac{\alpha}{2}\left(\mu+\frac{1}{2}\right)}}
$$




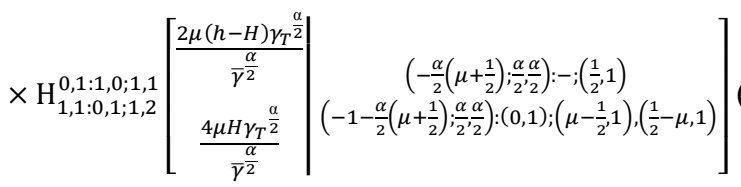

\section{Average Symbol Error Probability}

In this section, the ASEP for M-ary QAM signaling of a dual-branch SSC operating over a generalized $\alpha-\eta-\mu$ fading channel is derived. For M-QAM modulation scheme, the average SEP is obtained using the following averaging process [1]:

$$
\operatorname{Pr}(e)=\int_{0}^{\infty} P_{s}(E) f_{\gamma s s c}(\gamma) d \gamma
$$

where $P_{S}(E)$ is the conditional SEP for square M-QAM signals whose constellation size $M$ is given by $M=2^{k}$ with $k$ even. From 8.10 in $[1], P_{S}(E)$ is given by:

$$
P_{s}(E)=2 a \operatorname{erfc}(\sqrt{b \gamma})-a^{2} \operatorname{erfc}^{2}(\sqrt{b \gamma})
$$

where $\operatorname{erfc}(x)$ is the complementary error function, (i.e. $\left.\operatorname{erfc}(x)=\frac{2}{\sqrt{\pi}} \int_{x}^{\infty} e^{-t^{2}} d t,[1]\right), a=1-\frac{1}{\sqrt{M}}$ and $b=\frac{3 \log _{2}(M)}{2(M-1)}$.

Although (28) is obtained for square constellations, it gives a good approximation for $M$ for general QAM constellations with $M=2^{k}$ points which are either in the shape of a square $(k$ is even), or in the shape of a cross ( $k$ is odd) [1]. As a result, and after substituting (28) into (27), for M-QAM modulation scheme, the average SEP can be found as:

$$
\begin{gathered}
\operatorname{Pr}(e)=\left[1+F_{\gamma}\left(\gamma_{T}\right)\right] \\
\times(\underbrace{\int_{0}^{\infty} 2 a \operatorname{erfc}(\sqrt{b \gamma}) f_{\gamma}(\gamma) d \gamma}_{I_{3}}-\underbrace{\int_{0}^{\infty} a^{2} e r f c^{2}(\sqrt{b \gamma}) f_{\gamma}(\gamma) d \gamma}_{I^{\prime}}) \\
-(\underbrace{\int_{0}^{\gamma_{T}} 2 a \operatorname{erfc}(\sqrt{b \gamma}) f_{\gamma}(\gamma) d \gamma}_{I_{5}}-\underbrace{\int_{0}^{\gamma_{T}} a^{2} e r f c^{2}(\sqrt{b \gamma}) f_{\gamma}(\gamma) d \gamma}_{I_{6}})
\end{gathered}
$$

Differentiating (29) with respect to $\gamma_{T}$ and setting the result to zero [1], it can be shown that there is a generic expression for $\gamma_{T, o p t}$, for which the average error rate is minimal. In general, this $\gamma_{T, o p t}$ will be a solution of 9.254 in [1], but explicit closed-form solutions will not always be possible to obtain. In this case, one must rely on numerical root-finding techniques to find an accurate solution for the optimum threshold. To find the ASEP for M-QAM modulation scheme for SSC, we need to find the integrals, $I_{3}, I_{4}, I_{5}$ and $I_{6}$ in (29). These quantities are derived in terms of the BFHF as:

$$
\begin{aligned}
& I_{3}=\frac{2 a \alpha h^{\mu} \mu^{\mu+\frac{1}{2}}}{\sqrt{\pi} \Gamma(\mu) H^{\mu-\frac{1}{2}}(b \bar{\gamma})^{\frac{\alpha}{2}\left(\mu+\frac{1}{2}\right)}} \times \mathrm{H}_{2,1: 0,1 ; 1,2}^{0,2: 1,0 ; 1,1} \times \\
& {\left[\begin{array}{cc}
\frac{2 \mu(h-H)}{(b \bar{\gamma})^{\frac{\alpha}{2}}} & \left(\frac{1}{2}-\frac{\alpha}{2}\left(\mu+\frac{1}{2}\right) ; \frac{\alpha}{2}, \frac{\alpha}{2}\right),\left(1-\frac{\alpha}{2}\left(\mu+\frac{1}{2}\right) ; \frac{\alpha}{2}, \frac{\alpha}{2}\right):-;\left(\frac{1}{2}, 1\right) \\
\frac{4 \mu H}{(b \bar{\gamma})^{\frac{\alpha}{2}}} & \left(-\frac{\alpha}{2}\left(\mu+\frac{1}{2}\right) ; \frac{\alpha}{2}, \frac{\alpha}{2}\right):(0,1) ;\left(\mu-\frac{1}{2}, 1\right),\left(\frac{1}{2}-\mu, 1\right)
\end{array}\right]}
\end{aligned}
$$

$$
\begin{aligned}
& I_{4}=\frac{a^{2} \alpha h^{\mu} \mu^{\mu+\frac{1}{2}}}{\pi \Gamma(\mu) H^{\mu-\frac{1}{2}}(b \bar{\gamma})^{\frac{\alpha}{2}\left(\mu+\frac{1}{2}\right)}} \times H_{2,1: 0,1 ; 1,2 ; 1,2}^{0,2: 1,0 ; 1,2,0} \times \\
& {\left[\begin{array}{c}
\frac{2 \mu(h-H)}{(b \bar{\gamma})^{\frac{\alpha}{2}}} \\
\frac{4 \mu H}{(b \bar{\gamma})^{\frac{\alpha}{2}}} \\
1
\end{array} \mid \begin{array}{c}
\left(1-\frac{\alpha}{2}\left(\mu+\frac{1}{2}\right) ; \frac{\alpha}{2}, \frac{\alpha}{2}, 1\right),\left(\frac{1}{2}-\frac{\alpha}{2}\left(\mu+\frac{1}{2}\right) ; \frac{\alpha}{2}, \frac{\alpha}{2}, 1\right):--;\left(\frac{1}{2}, 1\right) ;(1,1) \\
\left(-\frac{\alpha}{2}\left(\mu+\frac{1}{2}\right) ; \frac{\alpha}{2}, \frac{\alpha}{2}, 1\right):(0,1) ;\left(\mu-\frac{1}{2}, 1\right),\left(\frac{1}{2}-\mu, 1\right) ;(0,1),\left(\frac{1}{2}, 1\right)
\end{array}\right]}
\end{aligned}
$$

$$
I_{5}=\frac{2 a \alpha h^{\mu} \mu^{\mu+\frac{1}{2}} \gamma_{T}^{\frac{\alpha}{2}\left(\mu+\frac{1}{2}\right)}}{\sqrt{\pi} \Gamma(\mu) H^{\mu-\frac{1}{2}} \bar{\gamma}^{\frac{\alpha}{2}\left(\mu+\frac{1}{2}\right)}} \times H_{1,1: 0,1 ; 1,2 ; 1,2,2}^{0,1: 1,0,1,2,0} \times
$$$$
\left.\left[\begin{array}{c}
\frac{2 \mu(h-H) \gamma_{T} \frac{\alpha}{2}}{\bar{\gamma}^{\frac{\alpha}{2}}} \\
\frac{4 \mu H \gamma_{T}^{\frac{\alpha}{2}}}{\bar{\gamma}^{\frac{\alpha}{2}}} \\
b \gamma_{T}
\end{array}\right] \begin{array}{c}
\left(1-\frac{\alpha}{2}\left(\mu+\frac{1}{2}\right) ; \frac{\alpha}{2}, \frac{\alpha}{2}, 1\right):-;\left(\frac{1}{2}, 1\right) ;(1,1) \\
\left(-\frac{\alpha}{2}\left(\mu+\frac{1}{2}\right) \frac{\alpha}{2} \frac{\alpha}{2}, 1\right):(0,1) ;\left(\mu-\frac{1}{2}, 1\right),\left(\frac{1}{2}-\mu, 1\right) ;(0,1),\left(\frac{1}{2}, 1\right)
\end{array}\right]
$$

$$
\begin{aligned}
& I_{6}=\frac{a^{2} \alpha h^{\mu} \mu^{\mu+\frac{1}{2}} \gamma_{T^{2}}^{\frac{\alpha}{2}\left(\mu+\frac{1}{2}\right)}}{\pi \Gamma(\mu) H^{\mu-\frac{1}{2}} \bar{\gamma}^{\frac{\alpha}{2}\left(\mu+\frac{1}{2}\right)}} \times \mathrm{H}_{1,1: 0,1 ; 1,2 ; 1,2 ; 1,2}^{0,1: 1,0 ; 1,2,0 ; 2} \times
\end{aligned}
$$

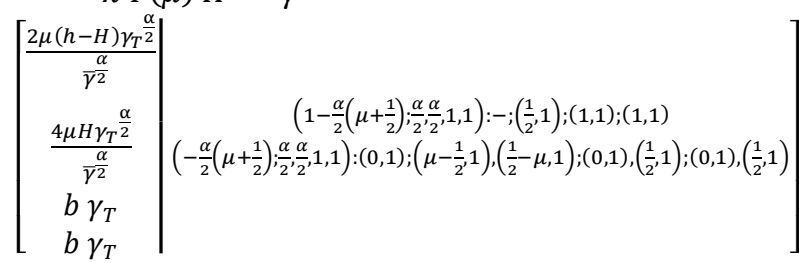

A closed form expression of the $\operatorname{Pr}(e)$ has been obtained, which is given as:

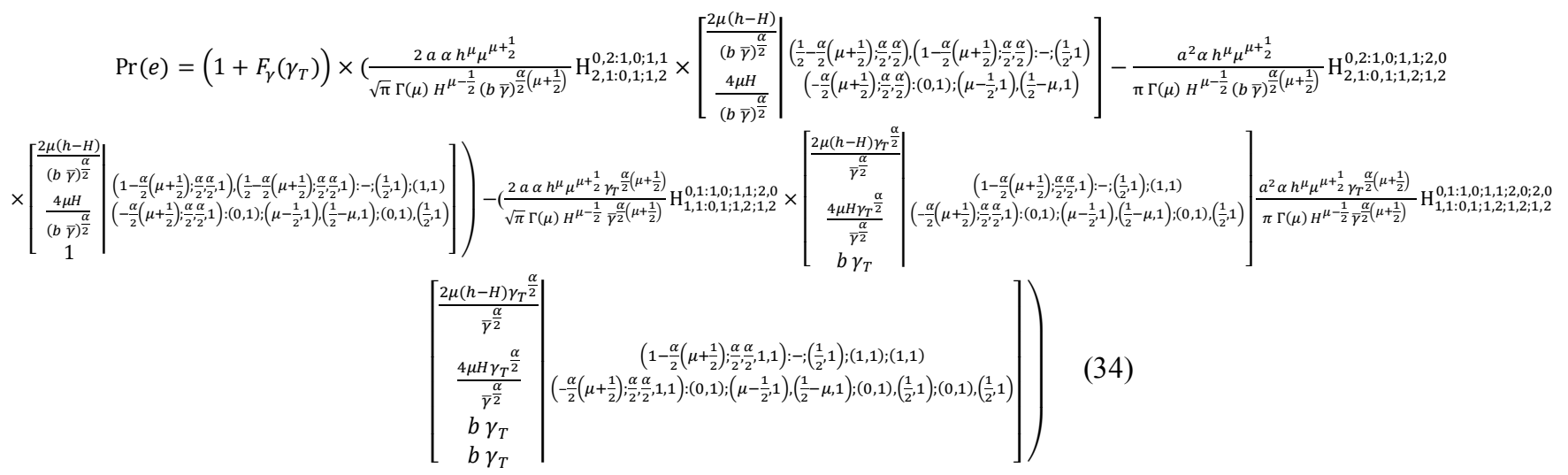




\section{RESULTS AND DISCUSSION}

In this section the $P_{\text {out }}$, ASNR and ASEP of a dual-branch SSC system over $\alpha-\eta-\mu$ fading channels are presented using several numerical examples. The results are obtained using (23), (26) and (34). The optimum switching threshold was applied in each example. To validate our results, we plot in Figures 1, 2 and 6, the ABEP for coherent BPSK and coherent BFSK, the $P_{\text {out }}$, and ASNR over $\eta-\mu$ and Hoyt (Nakagami- $q$ ), which are deduced from our results reported in $[6,12]$. The results exactly match the results reported in Figures 5 and 6 in [12], Figure 5 in [6], Figure 1 in [12], Figure 4 in [6], and Figure 2 in [12] respectively, which validates our work. The corresponding results for one-sided Gaussian and Nakagami- $m$ fading channels are presented as special cases of $\alpha-\eta-\mu$ fading channels. Figure 1 presents the ABEP for coherent BPSK and noncoherent BFSK for some special cases of fading channels. Figure 2 presents the outage probability with and without diversity over $\alpha-\eta-\mu$ fading channels vs the normalized outage threshold $\left(\gamma_{t h} / \bar{\gamma}\right)$ for different values of $\alpha, \eta$ and $\mu$. For $\alpha=2$, $\eta=0.5, \mu=0.5$ and $\left(\gamma_{t h} / \bar{\gamma}\right)=0 \mathrm{~dB}$, it is shown that $P_{\text {out }}^{S S C}\left(\gamma_{t h}\right)$ with diversity decreases (improves) by $41 \%$ when compared to the outage probability without diversity.

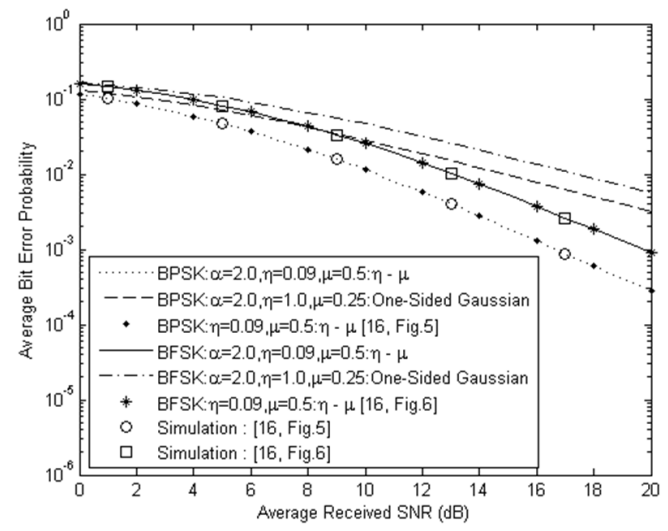

Fig. 1. ABEP for coherent BPSK and coherent BFSK for some special cases of fading channels

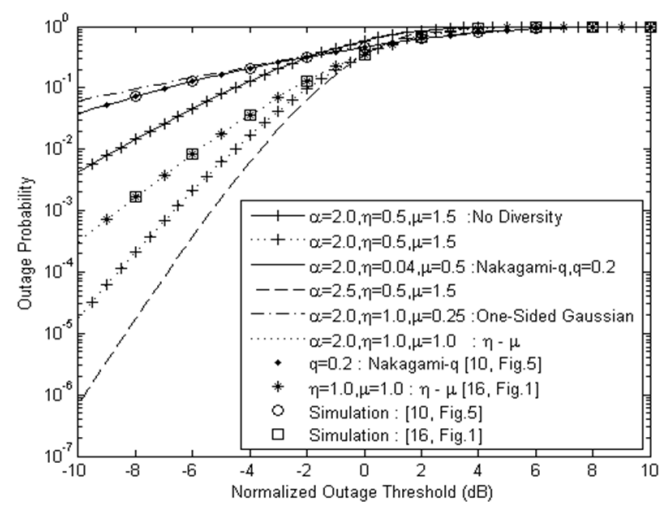

Fig. 2. Outage probability of a dual-branch SSC system over $\alpha-\eta-\mu$ fading channels versus normalized outage threshold $\left(\gamma_{t h} / \bar{\gamma}\right)$
Figures 3 and 4 show the effect of fading parameters on the outage probability $P_{\text {out }}^{s S c}\left(\gamma_{t h}\right)$ with average SNR $\bar{\gamma}=10 \mathrm{~dB}$ and $\gamma_{t h}=5 \mathrm{~dB}$. When $\alpha$ and (or) $\mu$ increase(s), they result in improved system performance. For example, in Figure 3, when $\eta=2$ (fixed) and $\mu=1.5$ (fixed), the $P_{\text {out }}^{s s c}\left(\gamma_{t h}\right)$ is approximately decreased by $54 \%$ when $\alpha$ is decreased from 1.1 to 0.7 . In Figure 4, when $\alpha=1.5$ (fixed) and $\eta=0.9$ (fixed), $P_{o u t}^{s s c}\left(\gamma_{t h}\right)$ is approximately decreased by $57 \%$ when $\mu$ is decreased from 1.5 to 1 . Also, as $\eta$ increases, $P_{\text {out }}^{\text {ssc }}\left(\gamma_{t h}\right)$ increases. For example, in Figure 3, when $\alpha=2$ (fixed) and $\mu=1.5$ (fixed), $P_{o u t}^{s s c}\left(\gamma_{t h}\right)$ is approximately increased by $31 \%$ when $\eta$ decreases from 2.5 to 1.5. This occurs for higher values of $\eta$. It is clear from the above discussion that $P_{\text {out }}^{s S C}\left(\gamma_{t h}\right)$ improves by increasing $\alpha$ and $\mu$, and degrades by increasing $\eta$, because of the following reasons: Parameter $\mu$ represents the multipath in each cluster, so, when $\mu$ increases the receiver will have more copies of the same transmitted signal, so $P_{o u t}^{s S C}\left(\gamma_{t h}\right)$ improves. Parameter $\alpha$ represents the power exponent of the sum of multipath components, therefore, as $\alpha$ increases, $P_{\text {out }}^{s s c}\left(\gamma_{t h}\right)$ improves. The fading parameter $\eta$ represents the correlation coefficient between the in-phase and quadrature components of each cluster of multipath. Therefore, as $\eta$ increases the correlation coefficient increases, and then the $P_{o u t}^{s S C}\left(\gamma_{t h}\right)$ degrades.

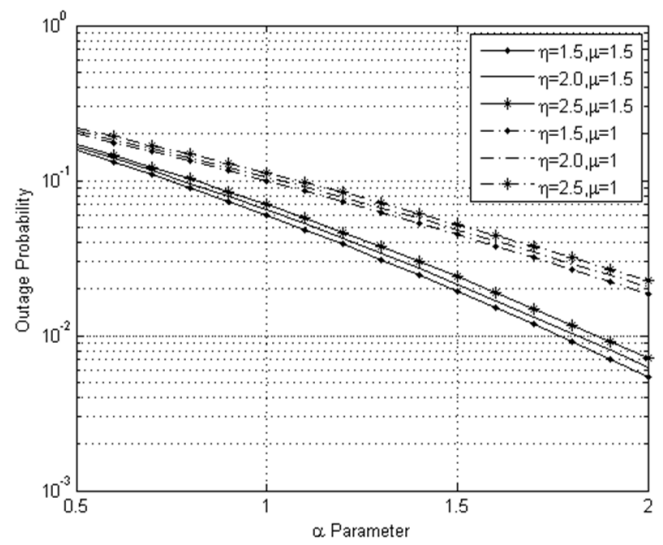

Fig. 3. Outage probability of a dual-branch SSC system over $\alpha-\eta-\mu$ fading channels versus parameter $\alpha$

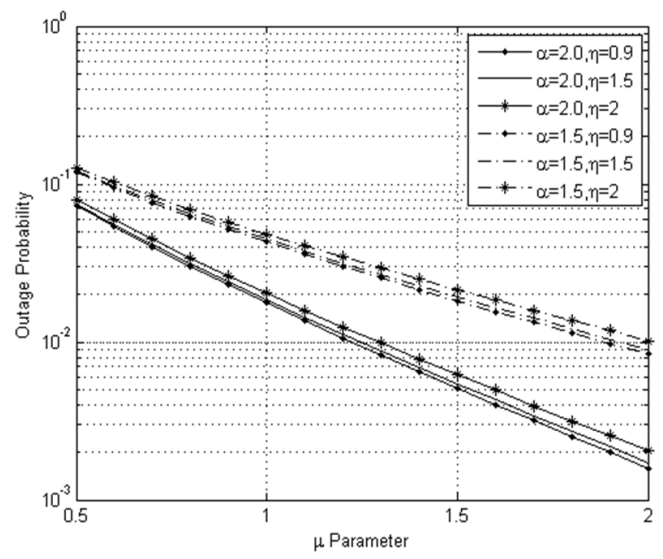

Fig. 4. Outage probability of a dual-branch SSC system over $\alpha-\eta-\mu$ fading channels versus parameter $\mu$ 
Figure 5 shows the normalized average output SNR of a dual-branch SSC system over fading channels $\left(\bar{\gamma}_{s s c} / \bar{\gamma}\right)$ versus the parameter $\alpha$ for different values of $\eta$ and $\mu$. Figure 6 shows the $\bar{\gamma}_{s S C} / \bar{\gamma}$ as a function of $\mu$ for different values of $\alpha$ and $\eta$. Both figures are plotted at $\gamma_{T}=0 \mathrm{~dB}$. The results presented in Figure 5 show that as $\alpha$ increases, the $\bar{\gamma}_{s s c} / \bar{\gamma}$ decreases, resulting in a reduced diversity gain. For example, in Figure 5, when $\eta=1.5$ (fixed) and $\mu=0.5$ (fixed), $\bar{\gamma}_{s s c} / \bar{\gamma}$ for $\alpha=1.4$ is approximately decreased by $7 \%$ compared to $\alpha=0.6$. We note similar observations for the effect of the fading parameter $\mu$ in Figure 6. The results indicate that as $\mu$ increases, the $\bar{\gamma}_{s s c} / \bar{\gamma}$ is degraded. For instance, when $\alpha=1.5$ (fixed) and $\eta=2.2$ (fixed), $\bar{\gamma}_{s S C} / \bar{\gamma}$ for $\mu=1.8$ is nearly decreased by $7.5 \%$ compared to $\mu=0.8$. In contrast to $\alpha$ and $\mu$, it is obvious from Figures 5 and 6 that as the value of $\eta$ increases, $\bar{\gamma}_{s s c} / \bar{\gamma}$ increases. For example, in Figure 6, when $\alpha=1$ (fixed) and $\mu=1.5$ (fixed), $\bar{\gamma}_{s s c} / \bar{\gamma}$ for $\eta=2.2$ is approximately increased by $1.5 \%$ compared to $\eta=1.2$. This effect of $\eta$ occurs for higher values of $\eta$.

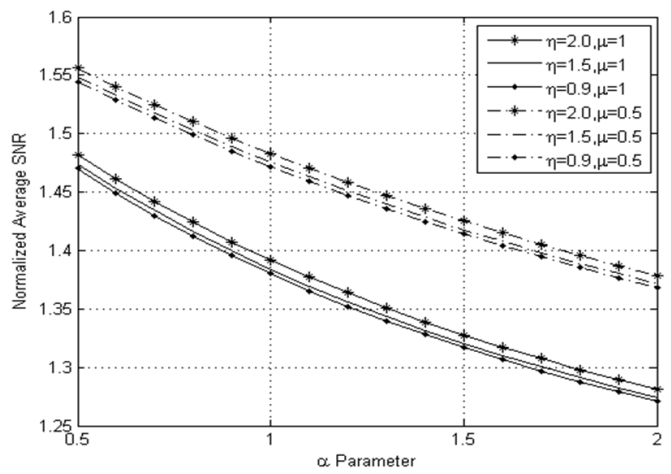

Fig. 5. Normalized average output SNR of a dual-branch SSC system over $\alpha-\eta-\mu$ fading channels $\left(\bar{\gamma}_{s S c} / \bar{\gamma}\right)$ versus parameter $\alpha$

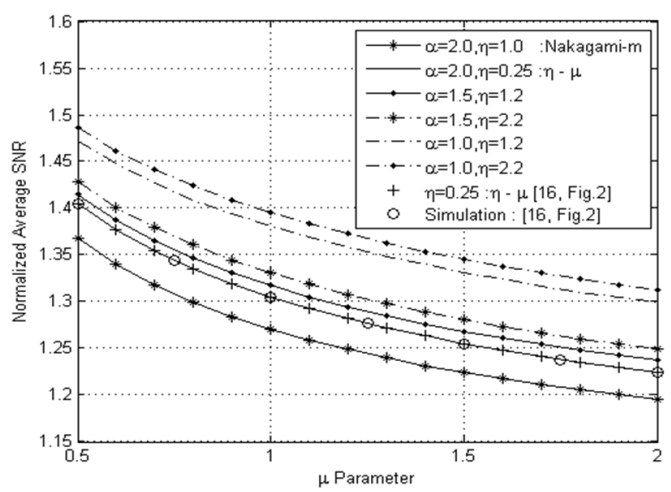

Fig. 6. Normalized average output SNR of a dual-branch SSC system over $\alpha-\eta-\mu$ fading channels $\left(\bar{\gamma}_{s s c} / \bar{\gamma}\right)$ versus parameter $\mu$

A graphical illustration of the impact of the switching threshold on $\bar{\gamma}_{s S c}$ for different values of $\alpha, \eta$ and $\mu$ is depicted in Figure 7. This Figure is plotted with respect to average SNR $\bar{\gamma}=10 \mathrm{~dB}$. Obviously, we get the best performance for $\gamma_{T}=\gamma_{T, o p t}=\bar{\gamma}$. Figure 8 shows the average SEP of 16QAM of a dual-branch SSC system over $\alpha-\eta-\mu$ fading channels versus the average $\operatorname{SNR}(\bar{\gamma})$ for different values of $\alpha, \eta$ and $\mu$, for switching threshold $\gamma_{T}=5 \mathrm{~dB}$. The curves in Figure 8 are categorized into different sets according to the average SNR value. Each combination of the parameters $\alpha, \eta$ and $\mu$ represents different channel model which justifies that each curve has different rate of change behavior within the same set. As expected, the ASEP performance improves as input branch SNR $\bar{\gamma}$ increases. The ASEP for a single branch $\alpha-\eta-\mu$ fading (no diversity) is also appeared in Figure 8. As shown, the system performance is improved under SSC diversity. For example, when $\alpha=1.5$ (fixed), $\eta=1.5$ (fixed), $\mu=2$ (fixed) and $\bar{\gamma}=10 \mathrm{~dB}$ (fixed), the ASEP under SSC diversity is approximately decreased by $59 \%$ compared to the ASEP for a single branch.

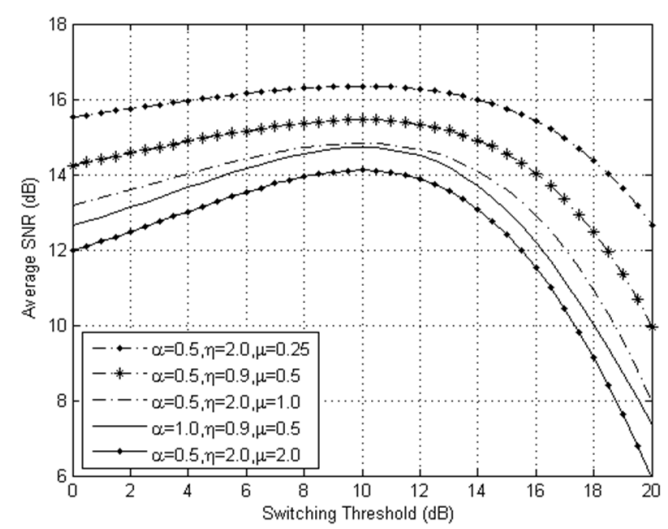

Fig. 7. Average output SNR of a dual-branch SSC system over $\alpha-\eta-\mu$ fading channels $\left(\bar{\gamma}_{S S C}\right)$ versus the switching threshold $\left(\gamma_{T}\right)$.

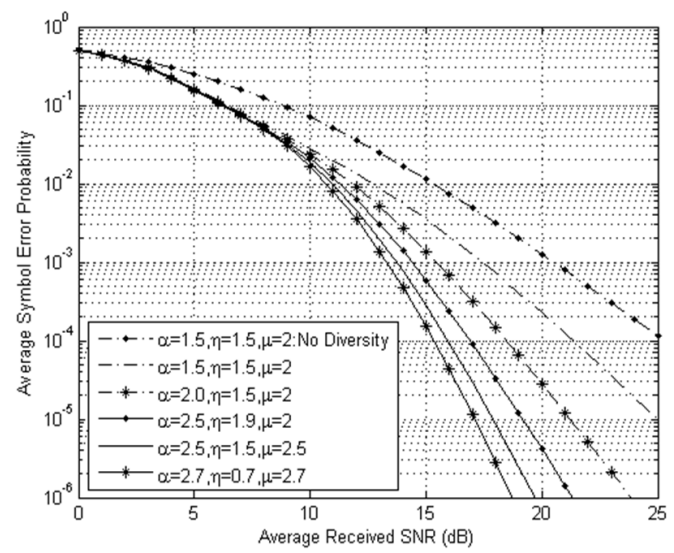

Fig. 8. ASEP of 16-QAM of a dual-branch SSC system over $\alpha-\eta-\mu$ fading channels versus average $\operatorname{SNR}(\bar{\gamma})$

Figures 9 and 10 show the effect of the fading parameters on the ASEP. The average SNR $\bar{\gamma}=10 \mathrm{~dB}$ and $\gamma_{T}=5 \mathrm{~dB}$. As $\alpha$ and (or) $\mu$ increase(s), the system performance improves. For example, in Figure 9, when $\eta=2$ (fixed) and $\mu=1$ (fixed), the ASEP reduction is about $39 \%$ when $\alpha$ increases from 0.6 to 0.95 . Figure 10 shows the effect of the parameter $\mu$ on the ASEP. For $\alpha=0.5$ (fixed) and $\eta=1.1$ (fixed), the ASEP reduction is about $33 \%$ when $\mu$ increases from 0.75 to 1.35 . In contrast with $\alpha$ and $\mu$, it is clear that as $\eta$ decreases, the ASEP improves. 
For instance, in Figure 9, when $\alpha=0.6$ (fixed) and $\mu=1$ (fixed), the ASEP reduction is about $10.5 \%$ when $\eta$ decreases from 2.5 to 0.9 . Note that, this effect occurs for higher values of $\eta$.

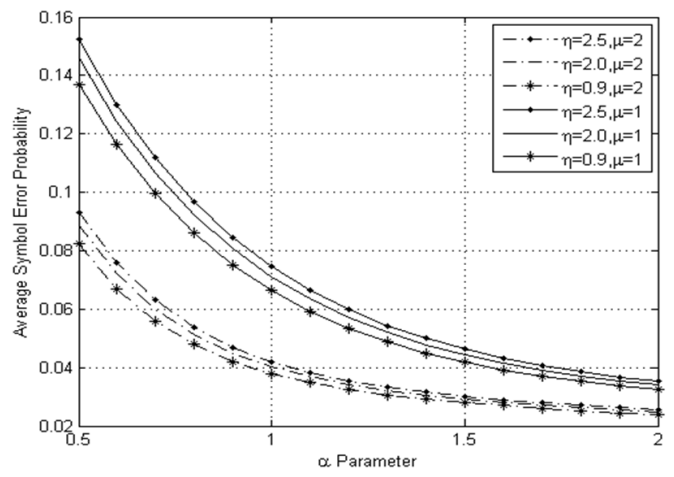

Fig. 9. ASEP of 16-QAM of a dual-branch SSC system over $\alpha-\eta-\mu$ fading channels versus $\alpha$

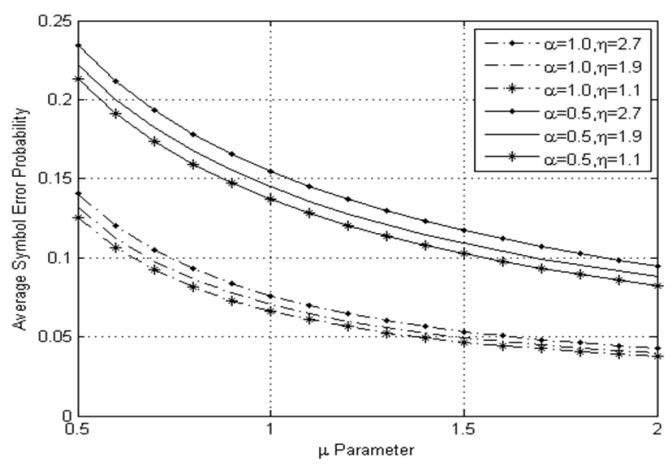

Fig. 10. ASEP of 16-QAM of a dual-branch SSC system over $\alpha-\eta-\mu$ fading channels versus $\mu$

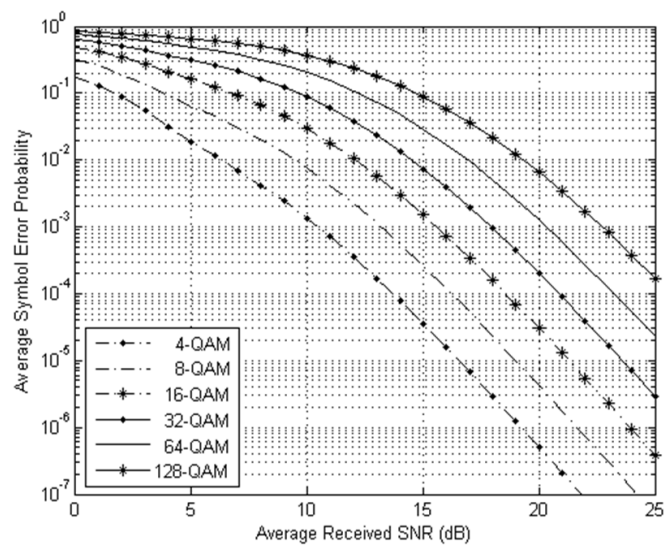

Fig. 11. ASEP of a dual-branch SSC system over $\alpha-\eta-\mu$ fading channels versus the average SNR $(\bar{\gamma})$ for multi levels of QAM

In Figure 11, we plot the ASEP of a dual-branch SSC system over $\alpha-\eta-\mu$ fading channels versus the average SNR $(\bar{\gamma})$ for multi levels of QAM: 4, 8, 16, 32, 64 and 128. Figure 11 is plotted for fixed values of $\alpha, \eta$ and $\mu(\alpha=2, \eta=1.5$ and $\mu=2$ ), and for $\gamma_{T}=3 \mathrm{~dB}$. The degradation in the ASEP is obvious with increasing values of M-ary of QAM. The ASEP get an improvement with increasing the average $\operatorname{SNR}(\bar{\gamma})$.

\section{CONCLUSIONS}

In this paper, a dual-branch SSC diversity scheme operating over $\alpha-\eta-\mu$ fading channel has been examined. New and closed-form analytical expressions were derived for ASNR, MGF, $P_{\text {out }}$, and ASEP for M-ary QAM signaling. Expressions for the optimum adaptive switching thresholds were also derived. Some of these expressions were obtained in terms of the well-known bivariate Fox's H-function (BFHF). The results $\mathrm{P}$ are shown to match those previously reported in the literature for other channels models such as $\eta-\mu$ model as a special case, which confirms the validity of the obtained expressions. Using numerical examples, we observed that the dual-branch SSC system has improved the performance of $P_{\text {out }}$, ASNR, and ASEP for M-ary QAM signaling. The $P_{\text {out }}$ and ASNR of the SSC diversity system improve, as $\alpha$ and (or) $\mu$ increase(s), with $\eta$ kept constant. The ASNR also improves, as $\eta$ increases, with $\alpha$ and $\mu$ kept constant. However, increasing $\alpha$ and $\mu$ improves the system performance more than $\eta$.

\section{REFERENCES}

[1] M. K. Simon, M. S. Alouini, Digital communication over fading channels: A unified approach to performance analysis, John Wiley \& Sons, 2000

[2] A. A. A. Dayya, N. C. Beaulieu, "Analysis of switched diversity systems on generalized-fading channels", IEEE Transactions on Communications, Vol. 42, No. 11, pp. 2959-2966, 1994

[3] A. A. A. Dayya, N. C. Beaulieu, "Switched diversity on microcellular Rician channels", IEEE Transactions on Vehicular Technology, Vol. 43, No. 4, pp. 970-976, 1994

[4] S. Haghani, N. C. Beaulieu, "Post detection switch-and-stay combining in Nakagami-m fading", IEEE Vehicular Technology Conference, Los Angeles, USA, September 26-29, 2004

[5] S. Haghani, N. C. Beaulieu, "Revised analyses of postdetection switchand-stay diversity in Rician fading", IEEE Transactions on Communications, Vol. 54, No. 7, pp. 1175-1178, 2006

[6] A. Chandra, C. Bose, M. K. Bose, "Performance of non-coherent MFSK with selection and switched diversity over Hoyt fading channel", Wireless Personal Communications, Vol. 68, No. 2, pp. 379-399, 2013

[7] P. S. Bithas, P. T. Mathiopoulos, "Performance analysis of SSC diversity receivers over correlated Rician fading satellite channels", EURASIP Journal on Wireless Communications and Networking, Vol. 2007, Article ID 25361, 2007

[8] P. S. Bithas, P. T. Mathiopoulos, G. K. Karagiannidis, "Switched diversity receivers over correlated Weibull fading channels", International Workshop on Satellite and Space Communications, Madrid, Spain, September 14-15, 2006

[9] S. Khatalin, J. P. Fonseka, "Capacity of correlated Nakagami-m fading channels with diversity combining techniques", IEEE Transactions on Vehicular Technology, Vol. 55, No. 1, pp. 142-150, 2006

[10] P. C. Spalevic, S. R. Panic, C. B. Dolicanin, M. C. Stefanovic, A. V. Mosic, "SSC diversity receiver over correlated $\alpha-\mu$ fading channels in the presence of cochannel interference", EURASIP Journal on Wireless Communications and Networking, Vol. 2010, Article ID 142392, 2010

[11] S. R. Panic, P. Spalevic, J. Anastasov, M. Stefanovic, M. Petrovic, "On the performance analysis of SIR-based SSC diversity over correlated $\alpha-\mu$ fading channels", Computers and Electrical Engineering, Vol. 37, No. 3, pp. 332-338, 2011 
[12] S. Khatalin, "On the performance analysis of SSC diversity system over $\eta-\mu$ fading channels", International Journal of Electronics, Vol. 103, No. 6, pp. 960-974, 2016

[13] S. Haghani, H. Dashtestani, "BER of noncoherent MFSK with post detection switch-and-stay combining in TWDP fading”, IEEE Vehicular Technology Conference, Quebec, Canada, September 3-6, 2012

[14] B. R. Manoj, P. R. Sahu, "Performance analysis of dual-switch and stay combiner over correlated KG fading channels", National Conference on Communications, New Delhi, India, February 15-17, 2013

[15] M. D. Yacoub, "The $\kappa-\mu$ distribution and the $\eta-\mu$ distribution", IEEE Antennas and Propagation Magazine, Vol. 49, No. 1, pp. 68-81, 2007

[16] Z. Hussain, A. R. Khan, H. Mehdi, S. M. A. Saleem, "Analysis of D2D communication system over $\kappa-\mu$ shadowed fading channel", Engineering, Technology \& Applied Science Research, Vol. 8, No. 5, pp. 3405-3410, 2018

[17] M. D. Yacoub, "The $\alpha-\mu$ distribution: A general fading distribution", $13^{\text {th }}$ IEEE International Symposium on Personal, Indoor and Mobile Radio Communications, Lisbon, Portugal, September 15-18, 2002

[18] G. Fraidenraich, M. D. Yacoub, "The $\alpha-\eta-\mu$ and $\alpha-\kappa-\mu$ fading distribution", IEEE 9th International Symposium on Spread Spectrum Techniques and Applications, Manaus-Amazon, Brazil, August 28-31, 2006

[19] G. Stamenovic, S. R. Panic, D. Rancic, C. Stefanovic, M. Stefanovic, "Performance analysis of wireless communication system in general fading environment subjected to shadowing and interference", EURASIP Journal on Wireless Communication and Networking, Vol. 124, pp. $1-8,2014$

[20] S. R. Panic, S. Ninkovic, D. Jaksic, S. Jovkovic, B. Milosevic, "Performance analysis of wireless communication system over $\alpha-\eta-\mu$ fading channels in the presence of CCI", Infoteh-Jahorina, Vol. 12, pp. 395-398, 2013

[21] O. S. Badarneh, M. S. Aloqlah, "Performance analysis of digital communication systems over $\alpha-\eta-\mu$ fading channels", IEEE Transactions on Vehicular Technology, Vol. 65, No. 10, pp. 7972-7981, 2016

[22] A. K. Papazafeiropoulos, S. A. Kotsopoulos, "The $\alpha-\lambda-\mu$ and $\alpha-\eta-\mu$ small-scale general fading distributions: A unified approach", Wireless Personal Communications, Vol. 57, No. 4, pp. 735-751, 2011

[23] A. M. Magableh, M. M. Matalgah, "Moment generating function of the generalized $\alpha-\mu$ distribution with applications", IEEE Communications Letters, Vol. 13, No. 6, pp. 411-413, 2009

[24] P. Z. Peebles, Probability, random variables, and random signal principles, 4th edition, McGraw-Hill, 2000

[25] A. P. Prudnikov, Y. A. Brychkov, O. I. Marichev, Integrals and series: special functions, CRC Press, 1990

[26] A. Erdelyi, Higher transcendental functions, McGraw-Hill, 1953

[27] I. S. Gradshteyn, I. M. Ryzhik, Table of integrals, series and products, 7th edition, Academic Press, 2007

[28] A. M. Mathai, R. K. Saxena, H. J. Haubold, The H-function: Theory and application, Springer, 2010 